\title{
Solutions for Linear Conservation Laws with Velocity Fields in $L^{\infty}$
}

\author{
Olivier Besson \& JÉrôme Pousin
}

Communicated by Y. BRENIER

\begin{abstract}
A Space-Time Integrated Least Squares (STILS) method is derived for solving the linear conservation law with a velocity field in $L^{\infty}$. An existence and uniqueness result is given for the solution of this equation. A maximum principle is established and finally a comparison with a renormalized solution is presented.
\end{abstract}

\section{Introduction}

Many works are dedicated to linear conservation laws and, according to the regularity of datum, different points of view have been used. The semi-group approach first developed in [6] requires a $C^{1}$ regularity for the velocity field. Moreover this vector field has to be extendable by zero outside a neighborhood of the spatial domain $\Omega$. The characteristic flow generated by the velocity $u$ can be defined for less regular fields. In [17], for a velocity field in $L^{1}\left(0, T ; W^{1,1}(\Omega)\right)$ with div $u \in L^{1}\left(0, T ; L^{\infty}(\Omega)\right)$, the notion of a renormalized solution is introduced allowing the handling of initial conditions with a very low regularity. When the velocity field $u$ belongs to $H^{1 / 2}$, with a divergence free existence and uniqueness, has been proved in [13]. When the velocity field $u$ belongs to $B V$, results of existence and uniqueness of solutions in $L^{\infty}$ is provided in [2, 12], see also [8]. For domains $\Omega$ included in $R^{2}$ and for a time independent velocity field $u$ in $L_{\text {Loc }}^{2}$, which is divergence free, a solution to the linear conservation laws is presented in [23] and compared to renormalized solutions. The question of uniqueness for weak solutions in $L^{\infty}$ to linear conservation laws is discussed in [15] for a velocity field $u$ in $L^{\infty}$ which is divergence free with a domain included in $\mathbb{R}^{2}$.

In this paper, the question of existence and uniqueness is addressed for linear conservation laws on a domain $\Omega$ with a Lipschitz boundary that satisfies the cone property. In our case the velocity field $u$ is only bounded, that is $u \in L^{\infty}$ and $\operatorname{div} u \in L^{\infty}$. The proposed method does not deal with the characteristic flow generated by the velocity field, but uses the functional setting of anisotropic Sobolev 
spaces in the same way as in [22] combined with a formulation of the problem in the sense of time-space least squares in the same spirit as in [1, 20]. In [17], the velocity field is required to be more regular than in our formulation $\left(u \in L^{\infty}\right.$, and $\left.\operatorname{div} u \in L^{\infty}\right)$. This allows the handling of boundary conditions with very few regularities. In our method we must assume that the boundary conditions have some regularity. The least squares method is widely used to solve partial differential equations, see $[18,20]$ for elasticity and fluid mechanics problems. Few general mathematical results have been obtained for this method in the case of first order time-dependent conservation laws. It seems that the STILS method (Space-Time Integrated Least Squares) originated in $[10,26]$. In [10, 28], a least squares method is used to solve a two-dimensional stationary first order conservation law with regularity assumptions on the advection velocity. Other results have been obtained in [3-5]. In this paper, a general mathematical analysis of this method is given for the linear conservation law when the advection velocity $u$ has low regularity, more precisely when $u \in L^{\infty}$, and $\operatorname{div} u \in L^{\infty}$. The solution obtained in this way is compared with weak and renormalized solutions [17].

In Section 2 a description of the problem is given. In Section 3 a variational formulation of the problem is given. Section 4 is dedicated to the proof of the existence and uniqueness of solutions to the variational formulation described in Section 3. Moreover a comparison with a renormalized solution is given.

\section{The problem description}

Let $\Omega \subset \mathbb{R}^{d}$ be a domain with a Lipschitz boundary $\partial \Omega$ satisfying the cone property. If $T>0$ is given, set $Q=\Omega \times] 0, T$ [. Consider an advection velocity $u: Q \rightarrow \mathbb{R}^{d}$ and $f \in L^{2}(Q)$ a given source term. Throughout this paper, the velocity $u$ has the following regularity

$$
u \in L^{\infty}(Q)^{d} \text { and } \operatorname{div} u \in L^{\infty}(Q) .
$$

Let

$$
\Gamma_{-}=\{x \in \partial \Omega:(u(x, t) \mid n(x))<0\},
$$

where $n(x)$ is the outer normal to $\partial \Omega$ at point $x$. For the sake of the presentation, it is assumed that $\Gamma_{-}$is not dependent on $t$.

The problem consists in finding a function $c: Q \rightarrow \mathbb{R}$ satisfying the following partial differential equation

$$
\partial_{t} c+\operatorname{div}(c u)=f \text { in } Q,
$$

and the initial and inflow boundary conditions

$$
\begin{array}{ll}
c(x, 0)=c_{0}(x) & \text { for } x \text { in } \Omega \\
c(x, t)=c_{1}(x, t) & \text { for } x \text { on } \Gamma_{-} .
\end{array}
$$

As usual, when $c_{1}, c_{0}$, and $u$ are sufficiently regular, changing the source term $f$ if necessary, one can assume that $c_{1}=0$ on $\Gamma_{-}$, and $c_{0}=0$ on $\Omega$. A similar result will be given later, using a suitable trace theorem. 


\section{Functional Setting}

In this section the functional setting for a variational formulation of the problem (2-4) is settled, (see also [3-5]). Moreover a trace operator is given in this context.

\subsection{The Hilbert spaces}

For $u \in L^{\infty}(Q)^{d}$, with div $u \in L^{\infty}(Q)$, define $\widetilde{u}$ as

$$
\widetilde{u}=\left(1, u_{1}, u_{2}, \ldots, u_{d}\right)^{t} \in L^{\infty}(Q)^{d+1}
$$

and for a sufficiently regular function $\varphi$ defined on $Q$, set

$$
\widetilde{\nabla} \varphi=\left(\frac{\partial \varphi}{\partial t}, \frac{\partial \varphi}{\partial x_{1}}, \frac{\partial \varphi}{\partial x_{2}}, \ldots, \frac{\partial \varphi}{\partial x_{d}}\right)^{t}
$$

and

$$
\widetilde{\operatorname{div}}(\widetilde{u} \varphi)=\frac{\partial \varphi}{\partial t}+\sum_{i=1}^{d} \frac{\partial}{\partial x_{i}}\left(u_{i} \varphi\right) .
$$

Finally $\widetilde{n}$ denotes the outward unit vector on $\partial Q$. The following theorem is proved in [11].

Theorem 1. Under the assumption $u \in L^{\infty}(Q)^{d}$, and $\operatorname{div} u \in L^{\infty}(Q)$, the normal trace of $u,(\widetilde{u} \mid \widetilde{n})$ is in $L^{\infty}(\partial Q)$.

Let now

$$
\begin{aligned}
\partial Q_{-} & =\{(x, t) \in \partial Q,(\widetilde{u} \mid \widetilde{n})<0\} \\
& =\Gamma_{-} \times(0, T) \cup \Omega \times\{0\},
\end{aligned}
$$

and set

$$
c_{b}(x, t)= \begin{cases}c_{0}(x) & \text { if }(x, t) \in \Omega \times\{0\} \\ c_{1}(x, t) & \text { if }(x, t) \in \Gamma_{-} \times(0, T) .\end{cases}
$$

For $\varphi \in \mathcal{D}(\bar{Q})$, consider the norm

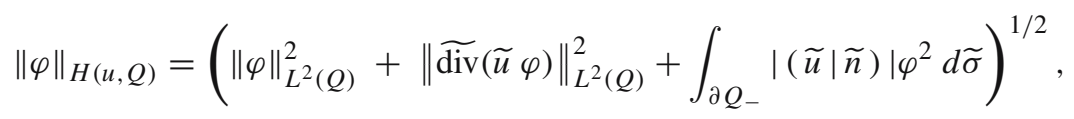

(see also $[3-5,7])$ and then define the space $H(u, Q)$ as the closure of $\mathcal{D}(\bar{Q})$ for this norm

$$
H(u, Q)=\overline{\mathcal{D}}(\bar{Q})^{H(u, Q)} .
$$

Set also

$$
W(u, Q)=\left\{\rho \in L^{2}(Q), \widetilde{\operatorname{div}}(\widetilde{u} \rho) \in L^{2}(Q),\left.\rho\right|_{\partial Q_{-}} \in L^{2}\left(\partial Q_{-},|(\widetilde{u} \mid \widetilde{n})| d \widetilde{\sigma}\right)\right\} .
$$

If $u$ is regular enough, it can be seen that

$$
H(u, Q) \cap L^{\infty}(Q)=W(u, Q) \cap L^{\infty}(Q),
$$

(see for example $[22,25])$. Indeed we have the following result. 
Theorem 2 (Meyers-Serrin Theorem). Let $u \in L^{2}\left(0, T ; H_{0}^{1}(\Omega)\right)$ with $\operatorname{div} u=0$. If $\rho \in L^{\infty}(Q)$ and $\widetilde{\operatorname{div}}(\widetilde{u} \rho) \in L^{2}(Q)$, then $\rho \in H(u, Q)$.

Let $\omega_{\epsilon}=\omega_{\epsilon}(x)$ (resp. $\left.\theta_{\epsilon}=\theta_{\epsilon}(t)\right)$ be the usual mollifier in $\mathbb{R}^{d}$ (resp. in $\mathbb{R}$ ). For the proof of Theorem 2, we need the following:

Proposition 1 (Commutation Theorem). Let $v \in H_{0}^{1}(\Omega)$ with, $\operatorname{div} v=0$; if $\phi \in L^{\infty}(\Omega)$ and $\operatorname{div}(v \phi) \in L^{2}(\Omega)$ then

$$
\left\|\operatorname{div}(v \phi) * \omega_{\epsilon}-\operatorname{div}\left(v\left(\phi * \omega_{\epsilon}\right)\right)\right\|_{L^{2}(\Omega)} \rightarrow 0
$$

when $\epsilon \rightarrow 0$.

Proof. Let $\bar{\phi}$ denote the zero extension of $\phi$ to the complement of $\Omega$. Then $\bar{\phi}$ verifies $\operatorname{div}(v \bar{\phi}) \in L^{2}\left(\mathbb{R}^{d}\right)$. The proof then proceeds in the same way as in [17] (Lemma II.1), [8] (Lemma 3.1) or [2] (Theorem 3.2). See also [19] (Lemma 2.5) for a proof when $v$ is $\mathcal{C}^{1}$.

Proof (of Theorem 2). Let us extend $\rho$ by 0 in $\mathbb{R}^{d}$ outside $\Omega$, the Lebesgue theorem implies that $\frac{\partial\left(\rho * \omega_{\epsilon}\right)}{\partial t} \in L^{2}\left(0, T ; L^{2}\left(\mathbb{R}^{d}\right)\right)$, so $\rho * \omega_{\epsilon} \in H^{1}\left(0, T ; L^{2}\left(\mathbb{R}^{d}\right)\right)$. Then, using the reflection extension principle in time, and keeping the same notations for simplicity, there is an extension $\rho * \omega_{\epsilon} \in H^{1}\left(\mathbb{R} ; L^{2}\left(\mathbb{R}^{d}\right)\right)$ of the initial function. Set now $\rho_{\epsilon}=\rho * \omega_{\epsilon} * \theta_{\epsilon}$, then $\rho_{\epsilon} \in \mathcal{D}(\bar{Q})$, and we have

$$
\begin{aligned}
\widetilde{\operatorname{div}}(\widetilde{u} \rho)-\widetilde{\operatorname{div}}\left(\widetilde{u} \rho_{\epsilon}\right)= & \widetilde{\operatorname{div}}(\widetilde{u} \rho)-\widetilde{\operatorname{div}}(\widetilde{u} \rho) * \omega_{\epsilon} * \theta_{\epsilon}+\widetilde{\operatorname{div}}(\widetilde{u} \rho) * \omega_{\epsilon} * \theta_{\epsilon} \\
& -\widetilde{\operatorname{div}}\left(\widetilde{u} \rho_{\epsilon}\right) .
\end{aligned}
$$

Clearly $\widetilde{\operatorname{div}}(\widetilde{u} \rho)-\widetilde{\operatorname{div}}(\widetilde{u} \rho) * \omega_{\epsilon} * \theta_{\epsilon}$ converges to zero in $L^{2}(Q)$ when $\epsilon \rightarrow 0$. Moreover

$$
\begin{aligned}
\widetilde{\operatorname{div}}(\widetilde{u} \rho) * \omega_{\epsilon} * \theta_{\epsilon}-\widetilde{\operatorname{div}}\left(\widetilde{u} \rho_{\epsilon}\right)= & \frac{\partial \rho}{\partial t} * \omega_{\epsilon} * \theta_{\epsilon}+\operatorname{div}(u \rho) * \omega_{\epsilon} * \theta_{\epsilon} \\
& -\frac{\partial \rho_{\epsilon}}{\partial t}-\operatorname{div}\left(u \rho_{\epsilon}\right) \\
= & \operatorname{div}(u \rho) * \omega_{\epsilon} * \theta_{\epsilon}-\operatorname{div}\left(u \rho_{\epsilon}\right) \\
= & \left(\operatorname{div}(u \rho) * \omega_{\epsilon}-\operatorname{div}\left(u\left(\rho * \omega_{\epsilon}\right)\right)\right) * \theta_{\epsilon} .
\end{aligned}
$$

So

$$
\begin{aligned}
& \left\|\widetilde{\operatorname{div}}(\widetilde{u} \rho) * \omega_{\epsilon} * \theta_{\epsilon}-\widetilde{\operatorname{div}}\left(\widetilde{u} \rho_{\epsilon}\right)\right\|_{L^{2}(Q)} \\
& \quad=\left\|\left(\operatorname{div}(u \rho) * \omega_{\epsilon}-\operatorname{div}\left(u\left(\rho * \omega_{\epsilon}\right)\right)\right) * \theta_{\epsilon}\right\|_{L^{2}(Q)} \\
& \quad \leq|\Omega|\left\|\left(\operatorname{div}(u \rho) * \omega_{\epsilon}-\operatorname{div}\left(u\left(\rho * \omega_{\epsilon}\right)\right)\right)\right\|_{L^{2}(Q)}
\end{aligned}
$$

and

$$
\begin{aligned}
& \left\|\left(\operatorname{div}(u \rho) * \omega_{\epsilon}-\operatorname{div}\left(u\left(\rho * \omega_{\epsilon}\right)\right)\right)\right\|_{L^{2}(Q)}^{2} \\
& =\int_{Q}\left\|\left(\operatorname{div}(u \rho) * \omega_{\epsilon}-\operatorname{div}\left(u\left(\rho * \omega_{\epsilon}\right)\right)\right)\right\|_{L^{2}(\Omega)}^{2} d t .
\end{aligned}
$$


Since

$$
\left\|\left(\operatorname{div}(u \rho) * \omega_{\epsilon}-\operatorname{div}\left(u\left(\rho * \omega_{\epsilon}\right)\right)\right)\right\|_{L^{2}(\Omega)}
$$

is bounded from above by an $L^{2}$ function, we get the result from Proposition 1 and the Lebesgue theorem.

We now give a trace result for functions belonging to $H(u, Q)$. Let us start with the well-known normal trace operator $\gamma$ defined from $H(\widetilde{\operatorname{div}}, Q)$ with values in $H^{-\frac{1}{2}}(\partial Q)($ see $[9,21])$

$$
\left.v \mapsto(\widetilde{n} \mid v)\right|_{\partial Q},
$$

for all $v \in H(\operatorname{div}, Q)$, with the associated Green formula

$$
\int_{Q} \widetilde{\operatorname{div}}(v) \psi+(v \mid \widetilde{\nabla} \psi) d x d t=<(v \mid \widetilde{n}), \psi>_{H^{-\frac{1}{2}}(\partial Q) ; H^{\frac{1}{2}}(\partial Q)},
$$

for all $\psi \in H^{1}(Q)$. Plugging $v=\widetilde{u} \rho$ in the previous formula, we have

$$
\int_{Q} \widetilde{\operatorname{div}}(\widetilde{u} \rho) \psi+(\widetilde{u} \mid \widetilde{\nabla} \psi) \rho d x d t=<\rho(\widetilde{u} \mid \widetilde{n}), \psi>_{H^{-\frac{1}{2}}(\partial Q) ; H^{\frac{1}{2}}(\partial Q)},
$$

for all $\psi \in H^{1}(Q)$. Let us now consider the bilinear form $L: \mathcal{D}(\bar{Q}) \times \mathcal{D}(\bar{Q}) \subset$ $H(u, Q) \times H(u, Q) \longrightarrow \mathbb{R}$ defined for all $\varphi, \psi \in \mathcal{D}(\bar{Q})$ by

$$
L(\varphi, \psi)=\int_{Q} \widetilde{\operatorname{div}}(\widetilde{u} \varphi) \psi+(\widetilde{u} \mid \widetilde{\nabla} \psi) \varphi d x d t+\int_{\partial Q_{-}}|(\widetilde{u} \mid \widetilde{n})| \varphi \psi d \widetilde{\sigma} .
$$

Accounting for Theorem 1 we have

$$
\begin{aligned}
|L(\varphi, \psi)| & \leqq\|\widetilde{\operatorname{div}}(\widetilde{u} \varphi)\|_{L^{2}(Q)}\|\psi\|_{L^{2}(Q)} \\
& +\|\widetilde{\operatorname{div}}(\widetilde{u} \psi)-\widetilde{\operatorname{div}}(\widetilde{u}) \psi\|_{L^{2}(Q)}\|\varphi\|_{L^{2}(Q)} \\
& +\|\varphi\|_{L^{2}\left(\partial Q_{-},|(\widetilde{u} \mid \widetilde{n})| d \widetilde{\sigma}\right)}\|\psi\|_{L^{2}\left(\partial Q_{-},|(\widetilde{u} \mid \widetilde{n})| d \widetilde{\sigma}\right)} .
\end{aligned}
$$

And the following estimate holds true

$$
|L(\varphi, \psi)| \leqq\left(1+\|\operatorname{div}(u)\|_{L^{\infty}(Q)}\right)\|\varphi\|_{H(u, Q)}\|\psi\|_{H(u, Q)} .
$$

Since it is straightforward to check that $L(\varphi, \varphi)=\|\varphi\|_{L^{2}\left(\partial Q_{+},|(\widetilde{u} \mid \widetilde{n})| d \widetilde{\sigma}\right)}^{2}$, if we extend by continuity the bilinear form $L$ to $H(u, Q) \times H(u, Q)$ we have:

Proposition 2. Under the assumption $u \in L^{\infty}(Q)^{d}$, and $\operatorname{div} u \in L^{\infty}(Q)$, there exists a linear continuous trace operator

$$
\begin{aligned}
\gamma_{\tilde{n}}: H(u, Q) & \longrightarrow L^{2}(\partial Q,|(\widetilde{u} \mid \tilde{n})| d \widetilde{\sigma}) \\
\varphi & \mapsto \gamma_{\tilde{n}} \varphi=\varphi_{\left.\right|_{\partial Q}},
\end{aligned}
$$

which can be localized as

$$
\begin{aligned}
\gamma_{\tilde{n}_{ \pm}}: H(u, Q) & \longrightarrow L^{2}\left(\partial Q_{ \pm},|(\widetilde{u} \mid \tilde{n})| d \widetilde{\sigma}\right) \\
\varphi & \mapsto \gamma_{\tilde{n}_{ \pm}} \varphi=\varphi_{\left.\right|_{\partial Q_{ \pm}} .}
\end{aligned}
$$


Finally define the spaces

$$
\begin{aligned}
H_{0}(u, Q) & =\left\{\rho \in H(u, Q), \rho=0 \text { on } \partial Q_{-}\right\}=H(u, Q) \cap \operatorname{Ker} \gamma \widetilde{n}_{-}, \\
V_{ \pm} & =\left\{\rho \in H(u, Q), \gamma_{\tilde{n}_{ \pm}} \rho \in L^{2}\left(\partial Q_{ \pm},|(\widetilde{u} \mid \tilde{n})| d \widetilde{\sigma}\right)\right\} \\
G_{ \pm} & =\gamma_{\tilde{n}_{ \pm}} V_{ \pm} .
\end{aligned}
$$

\subsection{Curved Poincaré inequality}

We now give an extension of the curved Poincaré inequality obtained in [3, 4].

Theorem 3. If $u \in L^{\infty}(Q)^{d}$ and $\operatorname{div} u \in L^{\infty}(Q)$, the semi-norm on $H(u, Q)$ defined by

$$
|\rho|_{1, u}=\left(\int_{Q}(\widetilde{\operatorname{div}}(\widetilde{u} \rho))^{2} d x d t+\int_{\partial Q_{-}}|(\widetilde{u} \mid \widetilde{n})| \rho^{2} d \widetilde{\sigma}\right)^{1 / 2}
$$

is a norm, equivalent to the norm given on $H(u, Q)$.

Proof. We have to show that there is a constant $C$ such that

$$
\|\varphi\|_{L^{2}(Q)} \leqq C \cdot|\varphi|_{1, u}
$$

for all $\varphi \in \mathcal{D}(\bar{Q})$. We have

$$
\begin{aligned}
& \int_{Q} {[\widetilde{\operatorname{div}}(\widetilde{u} \varphi) \cdot \xi+\varphi \cdot(\widetilde{u} \mid \widetilde{\nabla} \xi)] d x d t-\int_{\partial Q_{-}} \xi \varphi(\widetilde{u} \mid \widetilde{n}) d \widetilde{\sigma} } \\
& \quad=\int_{\partial Q_{+}} \xi \varphi(\widetilde{u} \mid \widetilde{n}) d \widetilde{\sigma}
\end{aligned}
$$

for all regular enough functions $\xi$. For $\alpha:(0, T) \rightarrow \mathbb{R}$, choose $\xi=\alpha \cdot \varphi$, then

$$
\begin{aligned}
\frac{\partial \xi}{\partial t}+(u \mid \nabla \xi) & =\alpha \cdot\left(\frac{\partial \varphi}{\partial t}+(u \mid \nabla \varphi)\right)+\alpha^{\prime} \varphi \\
& =\alpha \cdot\left(\frac{\partial \varphi}{\partial t}+\operatorname{div}(u \varphi)-\varphi \operatorname{div} u\right)+\alpha^{\prime} \varphi
\end{aligned}
$$

Let $v \in L^{\infty}(0, T)$ be defined by

$$
v(t)=\sup _{x \in \Omega}|\operatorname{div}(u(t, x))| .
$$

With the above choices, equation (7) has the form

$$
\begin{gathered}
\int_{Q}\left[\left(\alpha^{\prime}+\alpha v-\alpha(v+\operatorname{div} u)\right) \varphi^{2}+2 \alpha \varphi \cdot \widetilde{\operatorname{div}}(\widetilde{u} \varphi)\right] d x d t \\
-\int_{\partial Q_{-}} \alpha \varphi^{2}(\widetilde{u} \mid \tilde{n}) d \widetilde{\sigma}=\int_{\partial Q_{+}} \alpha \varphi^{2}(\widetilde{u} \mid \tilde{n}) d \widetilde{\sigma} .
\end{gathered}
$$

Let $\alpha$ be the solution of the differential equation

$$
\alpha^{\prime}+\alpha v=-2, \quad \alpha(T)=0 .
$$


An easy computation gives

$$
\alpha(t)=2 e^{-w(t)} \int_{t}^{T} e^{w(s)} d s \geqq 0,
$$

with $w(t)=\int_{0}^{t} e^{v(s)} d s$. Introducing this value in equation (8) we obtain

$$
\begin{gathered}
\int_{Q}\left[-2 \varphi^{2}-\alpha(v+\operatorname{div} u) \varphi^{2}+2 \alpha \varphi \widetilde{\operatorname{div}}(\widetilde{u} \varphi)\right] d x d t \\
-\int_{\partial Q_{-}} \alpha \varphi^{2}(\widetilde{u} \mid \widetilde{n}) d \widetilde{\sigma}=\int_{\partial Q_{+}} \alpha \varphi^{2}(\widetilde{u} \mid \widetilde{n}) d \widetilde{\sigma} \geqq 0 .
\end{gathered}
$$

Hence

$$
\begin{aligned}
& \int_{Q} \varphi^{2} d x d t \leqq \int_{Q} \alpha \varphi \cdot \widetilde{\operatorname{div}}(\widetilde{u} \varphi) d x d t-\frac{1}{2} \int_{\partial Q_{-}} \alpha \varphi^{2}(\widetilde{u} \mid \widetilde{n}) d \widetilde{\sigma} \\
& \quad \leqq \frac{1}{2} \int_{Q} \varphi^{2} d x d t+\frac{1}{2} \int_{Q} \alpha^{2} \widetilde{\operatorname{div}}(\widetilde{u} \varphi)^{2} d x d t-\frac{1}{2} \int_{\partial Q_{-}} \alpha \varphi^{2}(\widetilde{u} \mid \tilde{n}) d \widetilde{\sigma}
\end{aligned}
$$

So

$$
\int_{Q} \varphi^{2} d x d t \leqq \int_{Q} \alpha^{2} \cdot \widetilde{\operatorname{div}}(\widetilde{u} \varphi)^{2} d x d t-\int_{\partial Q_{-}} \alpha \varphi^{2}(\widetilde{u} \mid \widetilde{n}) d \widetilde{\sigma}
$$

If $A=\max \left(\|\alpha\|_{L^{\infty}}^{2},\|\alpha\|_{L^{\infty}}\right)$, we get

$$
\int_{Q} \varphi^{2} d x d t \leqq A\left(\int_{Q} \widetilde{\operatorname{div}}(\widetilde{u} \varphi)^{2} d x d t-\int_{\partial Q_{-}} \varphi^{2}(\widetilde{u} \mid \widetilde{n}) d \widetilde{\sigma}\right),
$$

and the theorem is proved.

Henceforth the space $H(u, Q)$ is equipped with the norm $|\varphi|_{1, u}$.

Remark 1. a) Using the above result, if $c_{b}=0$, the semi-norm

$$
|\rho|_{1, u}=\left(\int_{Q}(\widetilde{\operatorname{div}}(\widetilde{u} \rho))^{2} d x d t\right)^{1 / 2}
$$

in a norm on $H_{0}(u, Q)$ which is equivalent to the usual norm on $H(u, Q)$.

b) As an easy consequence of the above arguments, for any $\rho \in H(u, Q)$, the norm defined by

$$
\|\rho\| \|=\left(\|\rho\|_{L^{2}(Q)}^{2}+\frac{1}{2} \int_{\partial Q_{+}}(\tilde{u} \mid \tilde{n})(T-t) \rho^{2} d \widetilde{\sigma}\right)^{1 / 2}
$$

verifies

$$
\|\rho\|_{L^{2}(Q)} \leqq\||\rho|\| \leqq \sqrt{A}|\rho|_{1, u} .
$$




\subsection{A weak formulation}

In $L^{2}(Q)$, a solution of equation (2) corresponds to a minimizer in $\left\{\varphi \in H(u, Q) ; \gamma_{n_{-}}(\varphi)-c_{b}=0\right\}$ of the following convex, $H(u, Q)$-coercive functional

$$
J(c)=\frac{1}{2}\left(\int_{Q}(\widetilde{\operatorname{div}}(\widetilde{u} c)-f)^{2} d x d t-\int_{\partial Q_{-}} c^{2}(\widetilde{u} \mid \tilde{n}) d \widetilde{\sigma}\right) .
$$

The Gâteau derivative of $J$ is

$$
D J(c) \varphi=\int_{Q}(\widetilde{\operatorname{div}}(\widetilde{u} c)-f) \widetilde{\operatorname{div}}(\widetilde{u} \varphi) d x d t-\int_{\partial Q_{-}} c \varphi(\widetilde{u} \mid \widetilde{n}) d \widetilde{\sigma} .
$$

So a sufficient condition to get the least squares solution of (2-4) is the following weak formulation: if $c_{b} \in G_{-}$, find $c \in H(u, Q)$ such that

$$
\begin{aligned}
\int_{Q} \widetilde{\operatorname{div}}(\widetilde{u} c) \cdot \widetilde{\operatorname{div}}(\widetilde{u} \varphi) d x d t & =\int_{Q} f \cdot \widetilde{\operatorname{div}}(\widetilde{u} \varphi) d x d t \\
\gamma_{\tilde{n}_{-}}(c) & =c_{b}
\end{aligned}
$$

for all $\varphi \in H_{0}(u, Q)($ see $[3-5,7,14,16])$.

We give now a penalized formulation, useful for some $L^{\infty}$ estimate.

Lemma 1. If $c_{b} \in G_{-}$, let $c^{m}$ be the solution of

$$
\begin{aligned}
& \int_{Q} \widetilde{\operatorname{div}}\left(\widetilde{u} c^{m}\right) \cdot \widetilde{\operatorname{div}}(\widetilde{u} \varphi) d x d t-m \int_{\partial Q_{-}}\left(c^{m}-c_{b}\right) \cdot \varphi(\widetilde{u} \mid \widetilde{n}) d \widetilde{\sigma} \\
& \quad=\int_{Q} f \cdot \widetilde{\operatorname{div}}(\widetilde{u} \varphi) d x d t
\end{aligned}
$$

for all $\varphi \in H(u, Q)$. There is a subsequence of $c^{m}$ which weakly converges in $H(u, Q)$ to the solution $c$ of $(9)$.

Proof. Let $C_{b} \in V_{-}$with $\gamma \tilde{n}_{-} C_{b}=c_{b}$, and choose $\varphi=c^{m}-C_{b}$ in (10). We have the following a priori estimates

$$
\left\|\widetilde{\operatorname{div}}\left(\widetilde{u} c^{m}\right)\right\|_{L^{2}(Q)}^{2} \leqq\left\|\widetilde{\operatorname{div}}\left(\widetilde{u} C_{b}\right)\right\|_{L^{2}(Q)}^{2}+\|f\|_{L^{2}(Q)}^{2},
$$

and

$$
\int_{\partial Q_{-}}|(\widetilde{u} \mid \widetilde{n})|\left(c^{m}-c_{b}\right)^{2} d \widetilde{\sigma} \leqq \frac{1}{m}\left(\left\|\widetilde{\operatorname{div}}\left(\widetilde{u} C_{b}\right)\right\|_{L^{2}(Q)}^{2}+\|f\|_{L^{2}(Q)}^{2}\right) .
$$

Thus the sequence $c^{m}$ admits a subsequence, weakly converging to $c$ in $H(u, Q)$. Moreover $\gamma_{n_{-}} c=c_{b}$, so the limit in (10) is the solution of (9). 


\subsection{Stampacchia's theorems}

In this section, we assume that the domain $\Omega$ is bounded. Later we will use the following versions of Stampacchia's theorems (see [24, 27]).

Theorem 4. Let $\rho \in H(u, Q)$, then

$$
\widetilde{\operatorname{div}}(\widetilde{u} \rho)=0 \text { almost everwhere on the set }\{(x, t) \in Q ; \rho(x . t)=0\} .
$$

Theorem 5. Let $g: \mathbb{R} \rightarrow \mathbb{R}$ be a Lipschitz continuous function.

i) If $\rho \in H(u, Q)$, then $g(\rho) \in H(u, Q)$.

ii) If $g$ is differentiable except at a finite number of points, say $\left\{z_{1}, \ldots, z_{n}\right\}$, then

$$
\widetilde{\operatorname{div}}(\widetilde{u} g(\rho))=\left\{\begin{array}{l}
g^{\prime}(\rho) \widetilde{\operatorname{div}}(\widetilde{u} \rho)+g(\rho) \operatorname{div} u \text { if } \rho(x, t) \notin\left\{z_{1}, \ldots, z_{n}\right\}_{(12)} \\
0 \text { elsewhere. }
\end{array}\right.
$$

For the proof of these theorems the following lemma is used.

Lemma 2. Let $\rho \in H(u, Q)$ then $|\rho| \in H(u, Q)$ and

$$
\widetilde{\operatorname{div}}(\widetilde{u}|\rho|)=\operatorname{sgn}(\rho) \cdot \widetilde{\operatorname{div}}(\widetilde{u} \rho)+|\rho| \operatorname{div} u,
$$

where

$$
\operatorname{sgn}(\rho(x, t))=\left\{\begin{array}{cll}
+1 & \text { if } & \rho(x, t)>0 \\
0 & \text { if } & \rho(x, t)=0 \\
-1 & \text { if } & \rho(x, t)<0
\end{array}\right.
$$

Proof. (See [24]). For $\varepsilon>0$, let $g_{\varepsilon}(t)=\sqrt{t^{2}+\varepsilon}$. If $\rho \in H(u, Q)$, then $g_{\varepsilon}(\rho) \in H(u, Q)$ and

$$
\widetilde{\operatorname{div}}\left(\widetilde{u} g_{\varepsilon}(\rho)\right)=\frac{\rho}{\sqrt{\rho^{2}+\varepsilon}} \widetilde{\operatorname{div}}(\widetilde{u} \rho)+g_{\varepsilon}(\rho) \operatorname{div} u .
$$

We have

$$
\int_{Q}\left|g_{\varepsilon}(\rho)\right|^{2} d x d t=\varepsilon T|\Omega|+\int_{Q}|\rho|^{2} d x d t \rightarrow\|\rho\|_{L^{2}(Q)}^{2} \quad \text { if } \varepsilon \rightarrow 0
$$

and

$$
\begin{aligned}
\int_{Q}\left(g_{\varepsilon}^{\prime}(\rho) \widetilde{\operatorname{div}}(\widetilde{u} \rho)\right)^{2} d x d t & =\int_{Q} \frac{\rho^{2}}{\rho^{2}+\varepsilon} \widetilde{\operatorname{div}}(\widetilde{u} \rho)^{2} d x d t \\
& \rightarrow \int_{Q} \widetilde{\operatorname{div}}(\widetilde{u} \rho)^{2} d x d t \text { if } \varepsilon \rightarrow 0 .
\end{aligned}
$$

So the set $\left\{g_{\varepsilon}(\rho)\right\}_{\varepsilon>0}$ is bounded in $H(u, Q)$ and there exists a sequence $\left(\varepsilon_{n}\right) \rightarrow 0$ such that $g_{\varepsilon_{n}}(\rho) \rightarrow \eta$ in $H(u, Q)$. Since $\left\|g_{\varepsilon_{n}}(\rho)\right\|_{H(u, Q)} \rightarrow\|\rho\|_{H(u, Q)}$ if $n \rightarrow \infty$ and $g_{\varepsilon}(t) \rightarrow|\rho|$ if $\varepsilon \rightarrow 0$, we have $\eta=|\rho|$ and $|\rho| \in H(u, Q)$. 
Let now $\varphi \in \mathcal{D}(Q)$, then

$$
\begin{aligned}
\int_{Q} g_{\varepsilon}^{\prime}(\rho) \widetilde{\operatorname{div}}(\widetilde{u} \rho) \varphi d x d t & =\int_{Q}\left(\widetilde{\operatorname{div}}\left(\widetilde{u} g_{\varepsilon}(\rho)\right)-g_{\varepsilon}(\rho) \operatorname{div} u\right) \varphi d x d t \\
& \rightarrow \int_{Q}(\widetilde{\operatorname{div}}(\widetilde{u}|\rho|)-|\rho| \operatorname{div} u) \varphi d x d t
\end{aligned}
$$

But

$$
g_{\varepsilon}^{\prime}(\rho) \widetilde{\operatorname{div}}(\widetilde{u} \rho) \varphi \rightarrow \operatorname{sgn}(\rho) \widetilde{\operatorname{div}}(\widetilde{u} \rho) \varphi \text { a.e. }
$$

and

$$
\left|g_{\varepsilon}^{\prime}(\rho) \widetilde{\operatorname{div}}(\widetilde{u} \rho) \varphi\right| \leqq \operatorname{sgn}(\rho) \widetilde{\operatorname{div}}(\widetilde{u} \rho) \varphi
$$

and we get the second result.

Proof of Theorem 4. This is a consequence of Lemma 2. Indeed when $\rho \geqq 0$ then $|\rho|=\rho$ and

$$
\widetilde{\operatorname{div}}(\widetilde{u}|\rho|)=\widetilde{\operatorname{div}}(\widetilde{u} \rho)=\operatorname{sgn}(\rho) \widetilde{\operatorname{div}}(\widetilde{u} \rho)
$$

If $\rho=0$, then $\operatorname{sgn}(\rho)=0$, so $\widetilde{\operatorname{div}}(\widetilde{u} \rho)=0$ a.e. on the subset $\{(x, t) \in Q, \rho(x, t)=$ $0\}$. Let now $\rho \in H(u, Q)$, then $\rho^{+}=\frac{1}{2}(|\rho|+\rho) \in H(u, Q), \rho^{-}=\frac{1}{2}(|\rho|-\rho) \in$ $H(u, Q)$, and $\rho=\rho^{+}-\rho^{-}$. But

$$
\begin{aligned}
\{(x, t) \in Q, \rho(x, t) & =0\} \\
& =\left\{(x, t) \in Q, \rho^{+}(x, t)=0\right\} \cap\left\{(x, t) \in Q, \rho^{-}(x, t)=0\right\},
\end{aligned}
$$

so

$$
\widetilde{\operatorname{div}}\left(\widetilde{u} \rho^{+}\right)=0 \text { on }\left\{(x, t) \in Q, \rho^{+}(x, t)=0\right\},
$$

and

$$
\widetilde{\operatorname{div}}\left(\widetilde{u} \rho^{-}\right)=0 \text { on }\left\{(x, t) \in Q, \rho^{-}(x, t)=0\right\},
$$

and we get

$$
\widetilde{\operatorname{div}}(\widetilde{u} \rho)=0 \text { on }\{(x, t) \in Q, \rho(x, t)=0\},
$$

and the theorem is proved.

The proof of Theorem 5 is similar to the proof given in [24]. 


\section{Study of the least squares formulation}

This section is devoted to the study of equation (9). More precisely, an existence and uniqueness theorem for the solution of equation (9) is given. Then a maximum principle is deduced from Stampacchia's theorem.

Let us first show how to reduce the problem (2)-(4) to an homogeneous Dirichlet problem on $\partial Q_{-}$. For $c_{b} \in G_{-}$, let $C_{b} \in H(u, Q)$ be such that $\gamma_{\tilde{n}_{-}}\left(C_{b}\right)=c_{b}$. Then $\rho=c-C_{b}$ is the unique solution of

$$
\int_{Q} \widetilde{\operatorname{div}}(\widetilde{u} \rho) \cdot \widetilde{\operatorname{div}}(\widetilde{u} \psi) d x d t=\int_{Q}\left(f-\widetilde{\operatorname{div}}\left(\widetilde{u} C_{b}\right)\right) \cdot \widetilde{\operatorname{div}}(\widetilde{u} \psi) d x d t
$$

for all $\psi \in H_{0}(u, Q)$. Moreover the solution of problem (14) is equivalent to the solution of (2). Therefore, modifying the source term when necessary, it is sufficient to only deal with homogeneous Dirichlet boundary conditions on $\partial Q_{-}$.

With the notations and hypothesis of Section 3 we have

Theorem 6. For $u \in L^{\infty}(Q)^{d}$ with $\operatorname{div} u \in L^{\infty}(Q)$, and $c_{b} \in G_{-}$, and $f \in L^{2}(Q)$, the problem (14) has a unique solution. Moreover

$$
|\rho|_{1, u}=\|\widetilde{\operatorname{div}}(\widetilde{u} \rho)\|_{L^{2}(Q)} \leqq\|f\|_{L^{2}(Q)}+\left\|\widetilde{\operatorname{div}}\left(\widetilde{u} C_{b}\right)\right\|_{L^{2}(Q)},
$$

and the function $c=\rho+C_{b}$ is the space-time least squares solution of (2).

Proof. This assertion is a consequence of the curved Poincaré inequality (Theorem 3 ) and of the Lax-Milgram theorem (see also [3, 4]).

Remark 2. For the numerical solution of equation (14), a time marching approach can be used to avoid the consideration of all of $Q$ (see $[7,14,16])$.

Corollary 1. The solution c of equation (14) belongs to the space

$$
X=L^{2}(Q) \cap L^{2}\left(\partial Q_{+},(\widetilde{u} \mid \widetilde{n}) d \widetilde{\sigma}\right)
$$

equipped with the norm $\||c|||$.

The following theorem is a maximum principle for the solution of problem (14).

Theorem 7. Assume that the domain $\Omega$ is bounded, and the function $f=0$ in equation (9). Let $c_{b} \in G_{-} \cap L^{\infty}\left(\partial Q_{-}\right)$, if $\operatorname{div} u=0$, the solution $c$ of equation (9) satisfies

$$
\inf c_{b} \leqq c \leqq \sup c_{b}
$$

Proof. Let $c^{m}$ be the sequence of solutions to the penalized formulation given in Lemma 1. Then

$$
\begin{aligned}
& \int_{Q} \widetilde{\operatorname{div}}\left(\widetilde{u} c^{m}\right) \cdot \widetilde{\operatorname{div}}(\widetilde{u} \varphi) d x d t-m \int_{\partial Q_{-}} c^{m} \cdot \varphi(\widetilde{u} \mid \widetilde{n}) d \widetilde{\sigma} \\
& \quad=-m \int_{\partial Q_{-}} c_{b} \cdot \varphi(\widetilde{u} \mid \widetilde{n}) d \widetilde{\sigma}
\end{aligned}
$$


for all $\varphi \in H(u, Q)$. Set

$$
M=\sup _{\partial Q_{-}} c_{b}
$$

and put

$$
\varphi=\left(c^{m}-M\right)^{+}, Q_{1}=\left\{(x, t) \in \bar{Q}, c^{m}-M>0\right\}, \Sigma_{1}=\partial Q_{-} \cap Q_{1} .
$$

Then, from Stampacchia's lemma,

$$
\begin{aligned}
& \int_{Q_{1}} \widetilde{\operatorname{div}}\left(\widetilde{u} c^{m}\right) \cdot \widetilde{\operatorname{div}}\left(\widetilde{u}\left(c^{m}-M\right)\right) d x d t-m \int_{\Sigma_{1}} c^{m} \cdot\left(c^{m}-M\right)(\widetilde{u} \mid \widetilde{n}) d \widetilde{\sigma} \\
& \quad=-m \int_{\Sigma_{1}} c_{b} \cdot\left(c^{m}-M\right)(\widetilde{u} \mid \widetilde{n}) d \widetilde{\sigma} .
\end{aligned}
$$

Since div $u=0$,

$$
\begin{aligned}
& \int_{Q_{1}}\left(\widetilde{\operatorname{div}}\left(\widetilde{u}\left(c^{m}-M\right)\right)\right)^{2} d x d t-m \int_{\Sigma_{1}}\left(\left(c^{m}-M\right)\right)^{2}(\widetilde{u} \mid \tilde{n}) d \widetilde{\sigma} \\
& \quad=-m \int_{\Sigma_{1}}\left(c_{b}-M\right) \cdot\left(c^{m}-M\right)(\widetilde{u} \mid \widetilde{n}) d \widetilde{\sigma} \leqq 0 .
\end{aligned}
$$

Hence, using Theorem 3 , the set $Q_{1}$ has a zero measure, so $c^{m} \leqq M$. We show in the same way that $c^{m} \geqq \inf c_{b}$. Finally the conclusion holds for the weak limit $c$ of a subsequence of the sequence $c^{m}$.

\section{Comparison with weak and renormalized solutions}

This section is devoted to the comparison between the least squares solution of equation (2-4) and the renormalized solution of these equations in the sense of $[2,17]$.

More precisely, let $u \in L^{\infty}(Q)^{d} \cap L^{1}\left(0, T ; B V(\Omega)^{d}\right)$, with $\operatorname{div} u=0$, and $u=0$ on $\partial \Omega$. Let also $\phi_{b} \in G_{-} \cap L^{\infty}\left(\partial Q_{-}\right)$, and $\Phi_{b} \in H(u, Q)$ with $\phi_{b}=$ $\gamma \tilde{n}_{-}\left(\Phi_{b}\right)$, and finally set $\psi=-\widetilde{\operatorname{div}}\left(\widetilde{u} \Phi_{b}\right)+f \in L^{2}(Q)$. The space-time least squares solution $\rho \in L^{\infty}(Q)$ of

$$
\begin{aligned}
\partial_{t} \rho+\operatorname{div}(\rho u)=\psi & \text { in } Q, \\
\rho=0 & \text { on } \partial Q_{-},
\end{aligned}
$$

gives an equivalent solution to the previous problem (2-4).

Definition 1 [17]. For $u \in L^{\infty}(Q)^{d}$, div $u \in L^{\infty}(Q), c_{b} \in L^{\infty}\left(\partial Q_{-}\right)$, and $f \in L^{2}(Q)$, the function $c \in L^{\infty}(Q)$ is a renormalized solution of

$$
\widetilde{\operatorname{div}}(\widetilde{u} c)=f \quad \text { with } \quad c=c_{b} \quad \text { on } \partial Q_{-}
$$

if for any $\beta \in \mathcal{C}^{1}(\mathbb{R}), \beta(0)=0, \beta(c)$ is a weak solution of

$$
\widetilde{\operatorname{div}}(\widetilde{u} \beta(c))=\beta^{\prime}(c) f,
$$


and

$$
\beta(c)=\beta\left(c_{b}\right) \text { on } \partial Q_{-},
$$

where the equations are understood in the sense of distributions.

Let us first give some results concerning the operator

$$
\begin{aligned}
\mathcal{A}: H_{0}=H_{0}(u, Q) & \rightarrow L^{2}(Q) \\
\varphi & \mapsto \widetilde{\operatorname{div}}(\widetilde{u} \varphi) .
\end{aligned}
$$

As mentioned in Remark 1, the space $H_{0}$ is equipped with the the semi-norm

$$
|\rho|_{1, u}=\left(\int_{Q}(\mathcal{A} \rho)^{2} d x d t\right)^{1 / 2}
$$

which is equivalent to the usual norm.

Theorem 8. i) If $u \in L^{\infty}(Q)^{d}$, with $\operatorname{div} u \in L^{\infty}(Q)$, then

$$
\inf _{\varphi \in H_{0},\|\varphi\| \leqq 1} \sup _{\psi \in L^{2}(Q),\|\psi\| \leqq 1} \int_{Q} \widetilde{\operatorname{div}}(\widetilde{u} \varphi) \cdot \psi d x d t \geqq 1 .
$$

ii) If $u \in L^{\infty}(Q)^{d} \cap L^{1}\left(0, T ; B V(\Omega)^{d}\right)$, div $u=0$, then for all $\psi \in L^{\infty}(Q)$, $\psi \neq 0$,

$$
\sup _{\varphi \in H_{0},\|\varphi\| \leqq 1} \int_{Q} \widetilde{\operatorname{div}}(\widetilde{u} \varphi) \cdot \psi d x d t>0 .
$$

Proof. i) The inequality (17) is a straightforward consequence of Theorem 3.

ii) Let us prove (18); let $\psi \in L^{\infty}(Q)$, and assume that inequality (18) is false, then for all $\varphi \in \mathcal{D}(Q)$

$$
\int_{Q} \widetilde{\operatorname{div}}(\widetilde{u} \varphi) \cdot \psi d x d t=0
$$

Since

$$
\int_{Q} \widetilde{\operatorname{div}}(\widetilde{u} \varphi) \cdot \psi+\widetilde{\operatorname{div}}(\widetilde{u} \psi) \cdot \varphi d x d t=0
$$

we get $\widetilde{\operatorname{div}}(\widetilde{u} \psi)=0$.

Now let us show that $\psi=0$ on $\partial Q_{+}=\Omega \times\{T\}$. Let $\varphi \in \mathcal{D}(\bar{Q})$ with $\varphi=0$ on $\partial Q_{-}$, we have

$$
0=\int_{Q} \widetilde{\operatorname{div}}(\widetilde{u} \psi) \cdot \varphi d x d t=\int_{\partial Q_{+}}(\widetilde{u} \mid \widetilde{n}) \psi \varphi d \widetilde{\sigma}=\int_{\partial Q_{+}} \psi \varphi d \widetilde{\sigma} .
$$

Therefore the support of $(\tilde{u} \mid \tilde{n}) \psi$ is a subset of $\partial Q_{-}$, so $\psi_{\left.\right|_{\partial Q_{+}}}=0$. Hence $\psi$ satisfies

$$
\partial_{t} \psi+\operatorname{div}(u \psi)=0 \text { in } Q
$$


with the boundary condition

$$
\psi=0 \text { on } \partial Q_{+}=\Omega \times\{T\} .
$$

Let $\bar{u}$ denote the zero extension of $u$ to the complement of $\Omega$, then $\psi$ verifies

$$
\begin{aligned}
\partial_{t} \psi+\operatorname{div}(\bar{u} \psi)=0 & & \text { in } \mathbb{R}^{d} \times(0, T), \\
\psi=0 & & \text { on } \mathbb{R}^{d} \times\{T\} .
\end{aligned}
$$

For $\bar{u}:=-\bar{u} \in L^{1}\left(0, T ; B V(\Omega)^{d}\right)$, using [2], Theorem 3.5 and Theorem 4.1, equation (19-20) admits $\psi=0$ as a unique renormalized solution.

Corollary 2. The adjoint $\mathcal{A}^{*}$ of $\mathcal{A}: H_{0} \rightarrow L^{2}(Q)$ verifies

$$
\operatorname{ker} \mathcal{A}^{*} \cap L^{\infty}(Q)=\{0\} .
$$

So $L^{\infty}(Q) \subset R(\mathcal{A})$, the range of $\mathcal{A}$.

Proof. From Theorem 8 b), we have ker $\mathcal{A}^{*} \cap L^{\infty}(Q)=\{0\}$. Since $\mathcal{A}$ has a closed range, $L^{2}(Q)=\operatorname{ker} \mathcal{A}^{*}+R(\mathcal{A})$.

The next theorem shows that the solution of (9) is also a weak solution.

Theorem 9. For $f \in L^{\infty}(Q)$ and $u \in L^{\infty}(Q)^{d} \cap L^{1}\left(0, T ; B V(\Omega)^{d}\right)$, with div $u=0$, let $c$ be the solution of

$$
\int_{Q}[\widetilde{\operatorname{div}}(\widetilde{u} c)-f] \cdot \widetilde{\operatorname{div}}(\widetilde{u} \phi) d x d t=0
$$

for all $\phi \in H_{0}(u, Q)$, i.e. the function $c$ is the least squares solution of

$$
\begin{aligned}
\widetilde{\operatorname{div}}(\widetilde{u} c) & =f \\
c & =0 \quad \text { on } \partial Q_{-} .
\end{aligned}
$$

Then it is a weak solution

$$
\int_{Q}[\widetilde{\operatorname{div}}(\widetilde{u} c)-f] \cdot \phi d x d t=0
$$

for all $\phi \in L^{2}(Q)$.

Proof. Since $f \in L^{\infty}(Q)$, Corollary 2 gives the existence of $c \in H_{0}(u, Q)$, solution to (23). This solution also solves (21) which has a unique solution. Thus the least square solution $c$ is also a weak solution of (22).

Let $c_{b} \in L^{\infty}\left(\partial Q_{-}\right) \cap G_{-}$, and assume that $c_{b}=\gamma_{n_{-}}\left(C_{b}\right)$ with $C_{b} \in W^{1, \infty}(Q)$. We have the following:

Theorem 10. For $u \in L^{\infty}(Q)^{d} \cap L^{1}\left(0, T ; B V(\Omega)^{d}\right)$, with $\operatorname{div} u=0$, the least squares solution of

$$
\widetilde{\operatorname{div}}(\widetilde{u} c)=0 \text { with } c=c_{b} \text { on } \partial Q_{-}
$$

is a renormalized solution. 
Proof. From Theorem 7, the least squares solution $c$ of equation (24) is in $L^{\infty}(Q)$. Put $\rho=c-C_{b}$. Then $\rho$ is the least squares solution of

$$
\widetilde{\operatorname{div}}(\widetilde{u} \rho)=-\widetilde{\operatorname{div}}\left(\widetilde{u} C_{b}\right)=f \quad \text { with } \rho=0 \text { on } \partial Q_{-} .
$$

From Theorem 9 we get

$$
\int_{Q}[\widetilde{\operatorname{div}}(\widetilde{u} \rho)-f] \cdot \phi d x d t=0
$$

for all $\phi \in L^{2}(Q)$. Therefore $\widetilde{\operatorname{div}}(\widetilde{u} \rho)=f$ in $L^{2}(Q)$, so $\widetilde{\operatorname{div}}(\widetilde{u} c)=0$ in $L^{2}(Q)$ and $c=c_{b}$ on $\partial Q_{-}$. Since $c \in L^{\infty}(Q)$ we get $\widetilde{\operatorname{div}}(\widetilde{u} \beta(c))=\beta^{\prime}(c) \widetilde{\operatorname{div}}(\widetilde{u} c)=0$ and $\beta(c)=\beta\left(c_{b}\right)$ on $\partial Q_{-}$.

\section{Conclusions and remarks}

We have shown in this paper that the conservation law

$$
\partial_{t} c+\operatorname{div}(c u)=f \text { in } Q,
$$

can be solved for irregular vector fields, using a very simple approach compared to the methods used in $[2,8,17]$.

Our method leads to some numerical schemes which are much simpler to use than the usual one (like the streamline diffusion method, the characteristics method, the discontinuous finite element method with flux limiter, etc ...). Some numerical examples are presented in $[7,14,16]$.

In [6], it is proved that the solution of equation 25 gives an isomorphism on $L^{2}(Q)$ when $u \in \mathcal{C}^{1}(\Omega)$ is independent of $t$. Our method still gives an isomorphism, but in some unusual spaces. It allows the solving of equation 25 with an irregular velocity field $u$. This situation was unknown until now.

Acknowledgements. JÉRÔME PousIN was partially supported by a grant: projet Thématiques prioritaires Mathématiques de la région Rhône-Alpes 2003-2005.

\section{References}

1. Agoshkov, V.: Boundary value problems for transport equations. Birkhäuser, 1998

2. Ambrosio, L.: Transport equation and Cauchy problem for $B V$ vector fields. Invent Math. 158, 227-260 (2004)

3. AzÉRAD, P.: Analyse des équations de Navier-Stokes en bassin peu profond et de l'équation de transport. PhD dissertation, Université de Neuchâtel, 1996

4. AzÉrad, P., Pousin, J.: Inégalité de Poincaré courbe pour le traitement variationnel de l'équation de transport. C. R. Math. Acad. Sci. Paris 322, 721-727 (1996)

5. Azérad, P., Perrochet, P., Pousin, J.: Space-time integrated least-squares: A simple, stable and precise finite element scheme to solve advection equations as if they were elliptic. (Ed. Снірот, M. et al) Progress in partial differential equations: the Metz. surveys 4. Proceedings of the conference given at the University of Metz, France during the 1994-95 'Metz Days'. Harlow, Longman. Pitman Res. Notes Math. Ser. 345, 161-174 (1996) 
6. Bardos, C.: Problèmes aux limites pour les équations aux dérivées partielles du premier ordre à coefficients réels; théorèmes d'approximation; applications à l'équation de transport. Ann. Sci. Ecole. Norm. Sup. (4) 3, 185-233 (1970)

7. Besson, O, De Montmollin, G.: Space-time integrated least squares: a time marching approach. Internat. J. Numer. Methods Fluids 44, 525-543 (2004)

8. Bouchut, F.: Renormalized solutions to the Vlasov Equation with coefficients of bounded variation. Arch. Ration. Mech. Anal. 157, 75-90 (2001)

9. Brezzi, F., Fortin, M.: Mixed and hybrid finite element methods. Springer-Verlag, 1991

10. Сhattot, J.J., Guiu-Roux, J., Lamine, J.: Numerical solution of first-order conservation equation by a least squares method. Internat. J. Numer. Methods Fluids 2, 209219 (1982)

11. Chen, G.-Q., Frid, H.: Divergence-measure fields and hyperbolic conservation laws. Arch. Ration. Mech. Anal. 147, 89-118 (1999)

12. Colombini, F., Lerner, N.: Uniqueness of continuous solutions for BV vector fields. Duke Math. J. 111, 357-384 (2002)

13. Colombini, F., Luo, T., Rauch, J.: Uniqueness and nonuniqueness for nonsmooth divergence free transport. Séminaire: Equations aux Dérives Partielles, 2002-2003. Ecole Polytech., Palaiseau, 2003

14. De Montmollin, G.: Méthode STILS pour l'équation de transport: comparaisons et analyses. Etude d'un modèle de fermeture pour la loi de Darcy. PhD dissertation, Université de Neuchâtel, 2001

15. Depauw, N.: Non unicité des solutions bornées pour un champ de vecteurs BV en dehors d'un hyperplan. C. R. Math. Acad. Sci. Paris 337, 249-252 (2003)

16. Diallo, O.: Modélisation et simulation numérique de résines réactives dans un milieu poreux. $\mathrm{PhD}$ dissertation, Université Claude Bernard Lyon 1, 2000

17. Diperna, R.T., Lions, P.L.: Ordinary differential equations, transport theory and Sobolev spaces. Invent. Math. 98, 511-547 (1989)

18. Franca, L.P., Stenberg, R.: Error analysis of Galerkin least squares methods for the elasticity equations. SIAM-J. Numer. Anal. 28, 1680-1697 (1991)

19. Golse, F.: Introduction aux EDP d'évolution, Chapitre 1: équations de transport. Cours Univ. Paris 6, 2003-2004

20. Glowinski, R.: Numerical Methods for Nonlinear Variational problems. SpringerVerlag, 1984

21. Girault, V., Raviart, P.-A.: Finite element methods for the Navier-Stokes equations. Springer-Verlag, 1986

22. Franchi, B., Serapioni, R., Serra Cassano, F.: Meyer-Serrin type theorems and relaxation of variational integrals depending on vectors fields. Houston J. Math. 22, 859-890 (1996)

23. Hauray, M.: On two-dimensional Hamiltonian transport equations with $L_{\text {loc }}^{p}$ coefficients. Ann. Inst. H. Poincaré Anal. Non Linéaire 20, 625-644 (2003)

24. Kesavan, S.: Topics in functional analysis and applications. John Wiley \& Sons, 1989

25. Lions, P.L.: Mathematical topics in fluid mechanics. Vol. 1. Oxford Science Publications. Oxford University Press, Oxford, 1996

26. Nguyen, N., Reynen, J.: A space-time least squares finite element scheme for advection- diffusion equations. Comput. Methods Appl. Mech. Engrg. 42, 331-342 (1984)

27. Stampacchia, G.: Equations elliptiques du second ordre à coefficients discontinus. Presses de l’Université de Montréal, 1966

28. Wilders, P.: On the accuracy of least squares finite elements for a first-order conservation equation. Internat. J. Numer. Methods Fluids 8, 957-964 (1988) 
Université de Neuchâtel, Institut de Mathématiques, 11 , rue E. Argand, 2009 Neuchâtel, Switzerland.

e-mail: Olivier.Besson@unine.ch

and

INSA Lyon,

Institut C. Jordan, UMR-CNRS 5208,

Bâtiment Léonard de Vinci, 20 av. Albert Einstein,

69621 Villeurbanne, France.

e-mail: Jerome.Pousin@insa-lyon.fr

(Accepted October 21, 2006)

Published online April 24, 2007 - (C) Springer-Verlag (2007) 